\title{
Building bone to reverse osteoporosis and repair fractures
}

\author{
Sundeep Khosla, ${ }^{1}$ Jennifer J. Westendorf, ${ }^{2}$ and Merry Jo Oursler ${ }^{1}$
}

${ }^{1}$ Endocrine Research Unit and ${ }^{2}$ Department of Orthopedic Surgery, College of Medicine, Mayo Clinic, Rochester, Minnesota, USA.

\begin{abstract}
An important, unfilled clinical need is the development of new approaches to improve fracture healing and to treat osteoporosis by increasing bone mass. Recombinant forms of bone morphogenetic protein 2 (BMP2) and BMP7 are FDA approved to promote spinal fusion and fracture healing, respectively, and the first FDA-approved anabolic drug for osteoporosis, parathyroid hormone, increases bone mass when administered intermittently but can only be given to patients in the US for two years. As we discuss here, the tremendous explosion over the last two decades in our fundamental understanding of the mechanisms of bone remodeling has led to the prospect of mechanismbased anabolic therapies for bone disorders.
\end{abstract}

There are currently a number of FDA-approved drugs for the prevention and treatment of osteoporosis that work by inhibiting bone resorption (i.e., they are anticatabolic). However, these compounds, which include bisphosphonates, calcitonin, and selective estrogen receptor modulators, only prevent further loss of bone; they do not stimulate new bone formation. The only FDAapproved compound capable of stimulating new bone formation (and thus reversing bone loss) is parathyroid hormone (PTH). However, PTH is a protein that needs to be administered subcutaneously, and patients generally prefer taking an oral medication; there are potential side effects (e.g., hypercalcemia and hypercalciuria) associated with its use; and the duration of treatment with this drug is limited to 18 months in Europe and 24 months in the US, because rodents administered high doses of PTH were shown to develop osteosarcomas (1). Thus, there is a clear clinical need to develop new bone anabolic agents (particularly small molecules that can be used orally), and understanding the molecular details of the pathways that control bone formation is critical for the development of novel approaches to reverse osteoporosis.

It might be possible to modulate these pathways not only for the treatment osteoporosis, but also to accelerate the healing of fractures and to treat the $5 \%-10 \%$ of fractures that fail to heal satisfactorily $(2,3)$. This Review focuses on current approaches, as well as those on the horizon, that have the potential to achieve these goals. Although the osteoblast, as the bone-forming cell, is the obvious target for agents that aim to mediate bone anabolism, other cells are now also being considered as therapeutic targets. For example, osteocytes, cells that have historically largely been ignored because they lie entombed in the bone matrix, and osteoclasts, the boneresorbing cells that have long been a target for agents that are anticatabolic, are now also potential targets for drugs that stimulate bone anabolism. The development of agents that target more than one of these cell types might be the most effective therapy, as there is some evidence to suggest that PTH utilizes all of the above cell types in achieving its anabolic effects on bone.

Nonstandard abbreviations used: BMP, bone morphogenetic protein; BMU, basic multicellular unit; BRC, bone remodeling compartment; Dkk1, dickkopf homolog 1 ; EphB4, EPH receptor B4; Fz, Frizzled; GSK3 $\beta$, glycogen synthase kinase 3 $\beta$; LRP, LDL receptor-related protein; OPG, osteoprotegerin; PTH, parathyroid hormone; Runx2, Runt-related transcription factor 2; SfrP, secreted frizzled-related protein.

Conflict of interest: The authors have declared that no conflict of interest exists. Citation for this article: J. Clin. Invest. 118:421-428 (2008). doi:10.1172/JCI33612.

\section{Bone remodeling: evolving concepts}

Although macroscopically the skeleton seems to be a static organ, it is an extremely dynamic tissue at the microscopic level. Its ability to sustain the tremendous loads placed on it in everyday life depend on, among other factors, being able to remodel and repair the constant microcracks that develop both in cancellous bone - the "spongy" bone present in the vertebrae, pelvis, and ends (metaphyses) of long bones - and in cortical bone - the compact bone present in the shafts (diaphyses) of the long bones and surrounding cancellous bone in the vertebrae and pelvis. Since remodeling sites in cancellous bone in the vertebrae and pelvis are close to red marrow, which is known to contain osteoprogenitor cells (4), whereas remodeling sites in cortical bone are distant from red marrow, it had been assumed that the mechanisms of bone remodeling were likely to be different in cancellous versus cortical bone. Specifically, the assumption was that the cells needed for bone remodeling traveled directly from the red marrow to bone surfaces in cancellous bone, whereas they accessed cortical bone via the vasculature (5). However, it now seems that the fundamental mechanisms of bone remodeling might be very similar in both bone compartments, occurring in what has been termed the basic multicellular unit (BMU), which comprises the osteoclasts, osteoblasts, and osteocytes within the bone-remodeling cavity (Figure 1). Although the existence of the BMU has been established for a long time, the intimate relationship between the BMU and the vasculature, particularly in cancellous bone, was less well appreciated. This intimate relationship was initially described by Burkhardt et al. (6) more than 20 years ago and analyzed in detail in subsequent studies by Hauge and colleagues (7). These investigators demonstrated that the cells in the BMU, even in cancellous bone, were not directly contiguous to the bone marrow, but rather they were covered by a "canopy" of cells (most probably bone-lining cells) that seem to be connected to bone-lining cells on the quiescent bone surface (7). In turn, these bone-lining cells on the quiescent bone surface are in communication with osteocytes embedded within the bone matrix (7). Penetrating the canopy of bone-lining cells, and presumably serving as a conduit for the cells needed in the BMU, are capillaries; thus, the work of Hauge et al. (7) placed the BMU (consisting of osteoclasts, osteoblasts, and osteocytes), both in cancellous (Figure 1A) and in cortical (Figure 1B) bone, within the bone remodeling compartment (BRC), which comprises the BMU, the canopy of bone-lining cells, and the associated capillaries (Figure 1C and depicted schematically in Figure 1D). 

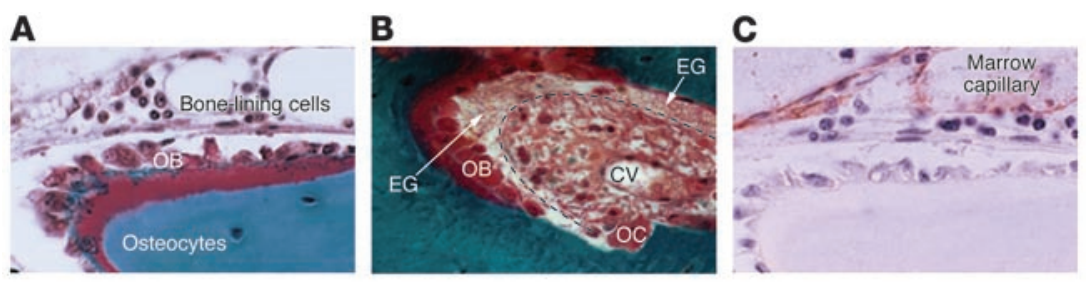

D

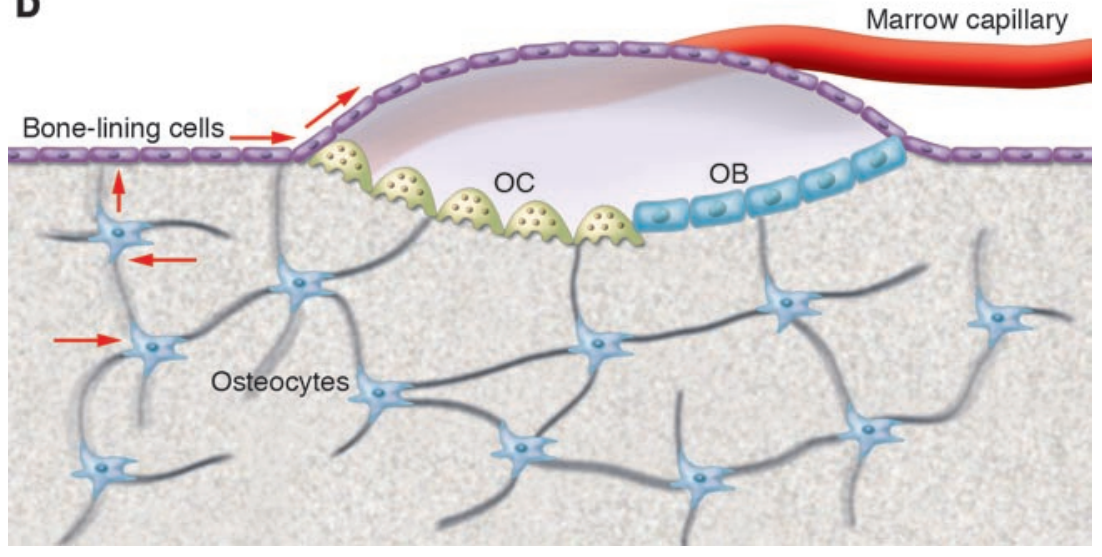

Figure 1

Histology (A-C; original magnification, $\times 40)$ and composite schematic (D) of the BRC, which comprises the cells constituting the BMU - specifically osteoclasts (OCs), osteoblasts (OBs), and osteocytes - as well as the canopy of bone-lining cells and the associated capillary. (A) BRC in cancellous bone, demonstrating the location of the OBs along the bone-forming surface. The osteocytes are shown embedded in the bone matrix and the canopy of cells consists of bone-lining cells. (B) BRC in cortical bone (outer demarcation indicated by the broken line) that is filled with erythrocyte ghosts (EG) and OBs; a few $\mathrm{OCs}$ are also seen. CV denotes the central vessel of the Haversian system, which forms the basic structural unit in cortical bone. (C) BRC stained with an antibody specific for CD34, which demonstrates staining of endothelial cells in the marrow capillary adjacent to the BRC. (D) Composite schematic of the BRC, showing connections between the osteocyte network, surface bone-lining cells, and the BRC. All cells in this network are connected with gap junctions, which might provide a pathway (arrows) by which signals generated by osteocytes deep within the bone reach the surface and elicit remodeling events by OCs and OBs. Note also the potential direct physical contact between OCs and OBs, which would allow for signaling between these cells. $\mathbf{A}$ and $\mathbf{C}$ are reproduced from Hauge et al. (7), and $\mathbf{B}$ and $\mathbf{D}$ from Eriksen et al. (10), with permission from the American Society for Bone and Mineral Research.

Given the structure of the BRC, it becomes easier to understand the key role that osteocytes have in controlling bone remodeling, despite being imprisoned in the bone matrix. It is now clear that osteocytes can sense microcracks and mechanical strain and be responsive to changes in the hormonal milieu of the bone (e.g., estrogen deficiency) to essentially "trigger" bone remodeling, perhaps by communicating with bone-lining cells $(8,9)$. The bone-lining cells, in turn, probably initiate the process of bone resorption (8), attract osteoclasts, and, together with adjoining bone-lining cells, begin to form the roof of the BRC. By analogy with remodeling in cortical bone, which is clearly associated with growth of a blood vessel into the remodeling site (5), the presumed ingrowth of a capillary into the BRC provides the vascular supply for the cells in the BMU of cancellous bone and might also provide the necessary osteoclasts and, subsequently, the osteoblasts that are needed for bone remodeling in both cancellous and cortical bone. Within the BMU, preosteoblastic cells, which express RANKL (10), probably control the differentiation of osteoclasts from hemato- poietic progenitors; in turn, completion of the bone resorption phase is followed by a wave of bone formation, driven, in part, by factors produced by the osteoclast that stimulate osteoblast differentiation and activity, perhaps via direct cell-cell contact between the osteoclast and osteoblast within the confines of the BRC. In the context of this orchestrated activity in the BMU, there are thus a number of cells that can be targeted by agents that increase bone mass: the osteoblast, the osteocyte, the osteoclast, and perhaps even the endothelial cell in the vasculature. Indeed, a recent study by Wang et al. (11) showed that increased VEGF production by osteoblastic cells has a marked anabolic effect on bone, apparently due to increased angiogenesis and a subsequent influx of osteoblasts onto bone surfaces. However, although the interactions of endothelial cells with osteoblasts is an active area of investigation (reviewed in ref. 12), a detailed discussion of this is beyond the scope of the present Review.

\section{Targeting osteoblast differentiation to enhance bone formation}

Overview of osteoblast differentiation. Numerous secreted factors of paracrine, autocrine, and endocrine origin influence osteoblast development and maturation. These include some bone morphogenetic proteins (BMPs), PTH, FGF, IGF, endothelin-1, and prostaglandin agonists $(13-16)$. Only a few are used clinically in the US to promote bone formation in response to injury or aging. Recombinant human BMP2 is used clinically to mediate spinal fusions, and BMP7 (also known as OP1) is used for the treatment of nonunion of long-bone fractures that occur secondary to trauma and for which an allograft is unsuitable. Neither is approved for osteoporosis therapy, because they both have a short half-life and cannot be administered systemically. As mentioned earlier, PTH is FDA approved to increase bone formation in patients with osteoporosis; however, it can only be prescribed for two years in the US. Thus, there is a clear need for more anabolic therapies to promote osteoblast activity. Targeting the Wnt pathway is one promising avenue. Available evidence indicates that Wnts and PTH stimulate bone formation via complementary pathways (17). BMP2 also synergizes with canonical Wnts to promote bone formation, and there seems to be considerable crosstalk between the Wnt and BMP signaling pathways $(18,19)$.

Osteoblasts are mesenchymal cells derived from mesodermal and neural crest progenitors. As cells of the osteoblast lineage differentiate, they produce molecules essential for construction of the mineralized bone matrix and for support of hematopoiesis and angiogenesis. Sequential expression of several molecules facilitates the differentiation of the progenitor cell into a proliferating preosteoblast, then into a bone matrix-producing osteoblast, and eventually into a mechanosensory osteocyte or a bone-lining cell (Figure 2). Runt-related transcription factor 2 (Runx2) is the earliest marker of an osteoblast lineage cell and is necessary, but not 


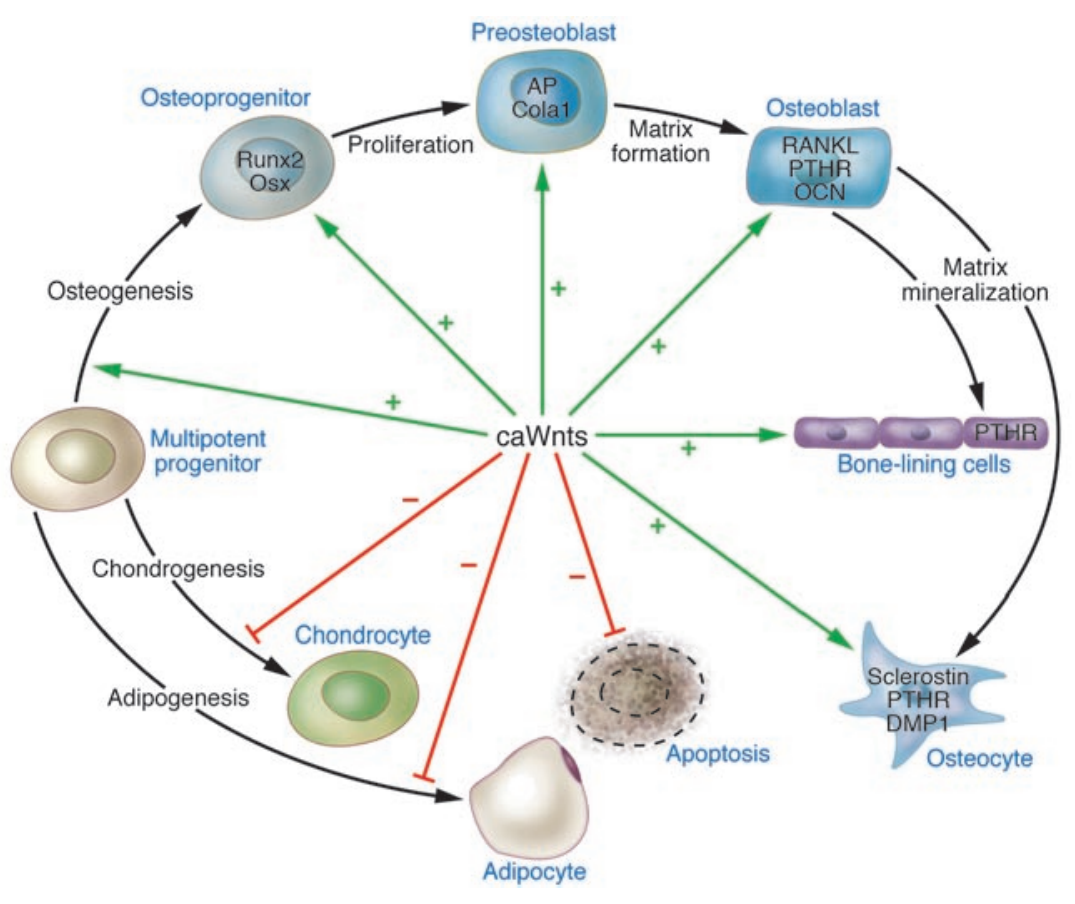

\section{Figure 2}

The central role of canonical Wnt signaling in regulating osteoblast lineage specification, expansion, and terminal differentiation. Osteoblasts are derived from multipotent mesodermal or neural crest progenitors. Activation of the canonical (ca) Wnt signaling pathway, manifest through $\beta$-catenin stabilization, prevents the formation of cartilage (chondrogenesis). Wnt10b prevents adipogenesis (40). The canonical Wnt signaling pathway promotes survival of all cells of the osteoblast lineage and induces the proliferation of preosteoblasts. + , canonical Wnt signaling promotes the process; -, canonical Wnt signaling inhibits the process; AP, alkaline phosphatase; Cola1, collagen $\alpha 1$; DMP1, dentin matrix protein 1; OCN, osteocalcin; Osx, osterix; PTHR, receptor for PTH. sufficient, for a progenitor cell to differentiate along the osteoblast lineage $(20,21)$. Runx 2 regulates cellular proliferation, integrates numerous signaling pathways, and controls the expression of many genes (e.g., those encoding osteocalcin, VEGF, RANKL, sclerostin, and dentin matrix protein 1 [DMP1]) throughout osteoblast maturation $(22,23)$. Osterix is another transcription factor essential for the formation of preosteoblasts from multipotent progenitors (24). It acts downstream of Runx2, but its expression is downregulated in bone marrow stromal cells by endothelial cells (25); osterix, in turn, impairs osteoblast differentiation (24). Osterix is also a suppressor of VEGF expression following endothelin-1 exposure (26); therefore, it might be a crucial mediator of osteoblast function within the context of the BRC. During the matrix-forming and maturation phases of osteoblast differentiation, the simultaneous coexpression of alkaline phosphatase and type I collagen sets the stage for mineralization (27). Mature osteoblasts also produce osteocalcin, RANKL, and the receptor for PTH (PTHR1), which, among other actions, regulate bone formation and resorption. As osteoblasts become embedded in the mineralized matrix, they transform into osteocytes and begin expressing several molecules, including DMP1 and sclerostin, that control bone formation and phosphate metabolism (28).

Wnts and osteoblast differentiation. Wnts are secreted glycoproteins crucial for the development and homeostatic renewal of many tissues, including bone. Figure 2 depicts the central role played by the canonical Wnt signaling pathway in regulating osteoblast development. Wnts stimulate several signaling pathways by binding a receptor complex consisting of LDL receptor-related protein 5 (LRP5) or LRP6 and one of ten Frizzled (Fz) molecules (18). The canonical Wnt signaling pathway has been the most extensively studied Wnt signaling pathway in osteoblasts. It involves the stabilization of $\beta$-catenin and regulation of multiple transcription factors, namely lymphoid enhancer factor 1 (Lef1) and the T cell factors Tcf7 (often referred to as Tcf1), Tcf7L1 (often referred to as Tcf3), and Tcf7L2 (often referred to as Tcf4) (Figure 3). This pathway is active in all cells of the osteoblast lineage, including preosteoblasts, bone-lining cells, and osteocytes (29). Notably, Wnt/ $\beta$-catenin signaling is a normal physiological response to mechanical loading (30) and participates in the fracture healing process (31). Wnt signaling has three major functions in osteoblast lineage cells: dictating osteoblast specification from osteo-/chondroprogenitors; stimulating osteoblast proliferation; and enhancing osteoblast and osteocyte survival. Within the BMU, Wnts influence osteoclast maturation by regulating RANKL levels in osteoblasts (32).

The crucial role of the canonical Wnt signaling pathway in bone cells was revealed earlier this decade in seminal studies showing that loss-of-function mutations in the gene encoding LRP5 decrease bone mass, whereas gain-of-function mutations increase bone mass in both humans and mice (33-36). The mouse models also revealed that $\operatorname{Lrp} 5$ is mechanistically essential for regulating osteoblast number, through both increasing the proliferation of progenitors and enhancing the survival of committed osteoblasts and osteocytes $(35,36)$. In accordance with these findings, adding Wnts or deleting secreted Wnt inhibitors enhances osteoblast and osteocyte survival (37-39). Since the LRP5 discoveries, many other components of the Wnt signaling pathway (e.g., Wnt10b, $\beta$-catenin, Tcf1, adenomatosis polyposis coli [APC], Axin-2, secreted frizzled-related protein 1 [Sfrp1], Sfrp4, and dickkopf homolog 1 [Dkk1]) have been deleted or overexpressed in mice. The general conclusion derived from all these studies is that activation of the canonical Wnt signaling pathway facilitates osteoblast specification from mesenchymal progenitors at the expense of adipogenesis and enhances bone mass and strength, whereas suppression causes bone loss $(38,40-50)$. Several of these studies also revealed that the canonical Wnt signaling pathway cooperates with the essential transcription factors Runx 2 and osterix to maintain and promote osteoblast maturation (41-43, 45, 50).

Although the final conclusion (that stimulation of the canonical Wnt signaling pathway increases bone mass) was similar in the above-mentioned studies (38, 40-50), the underlying mechanisms 


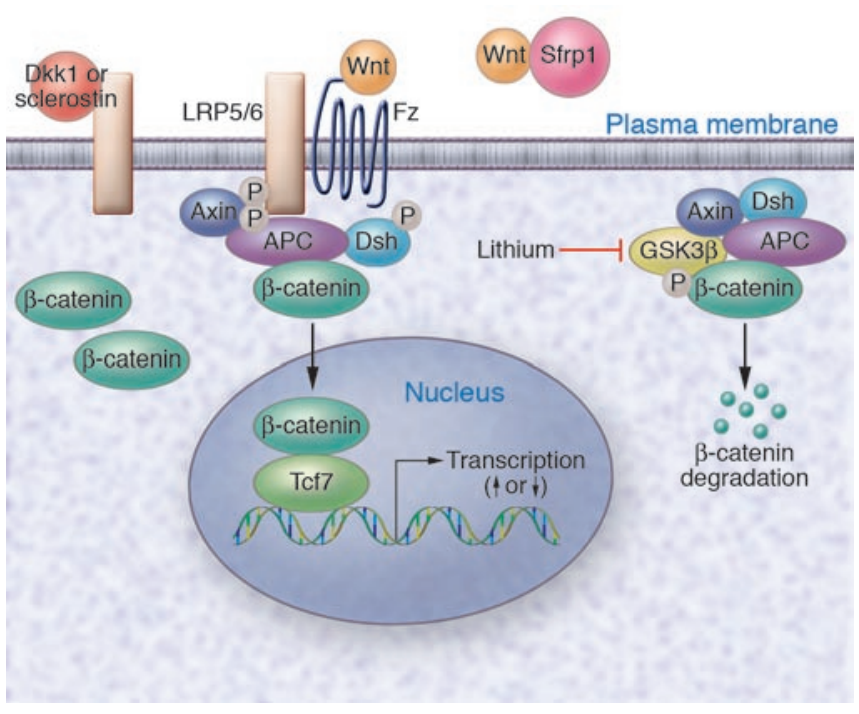

Figure 3

A partial view of the canonical Wnt signaling pathway. Wnts bind a receptor complex consisting of LRP5 or LRP6 and one of ten Fz proteins. This prevents phosphorylation of $\beta$-catenin by GSK3 $\beta$ and other kinases and its subsequent degradation. Of note, mutating the residues that can be phosphorylated to alanine creates stable, gainof-function $\beta$-catenin proteins. Stabilized $\beta$-catenin accumulates and translocates to the nucleus, where it interacts with Tcf7 and related transcription factors (Lef1, Tcf7L1, Tcf7L2) to regulate gene expression. Outside the cell, molecules that sequester either LRP5 (e.g., Dkk1 and sclerostin) or the Wnt ligand (e.g., Sfrp) negatively control the canonical Wnt signaling pathway. Lithium chloride inhibits GSK3 $\beta$ inside the cell. APC, adenomatosis polyposis coli.

sometimes differed. For example, constitutively active $\beta$-catenin increases bone mass by inducing the expression of the gene encoding osteoprotegerin (OPG) and thereby inhibits osteoclast maturation $(41,50)$. In contrast, $\operatorname{Lrp} 5$ gain-of-function mutations or administration of Wnt3a enhance the proliferation of preosteoblasts and prevent apoptosis of osteoblasts and osteocytes without affecting osteoclasts $(35,37,51,52)$. Thus, activation of Wnt signaling pathways through Wnt proteins binding to their receptors triggers different signaling pathways than if $\beta$-catenin is the starting point (37). $\beta$-Catenin participates in other signaling pathways, including ones induced by activated receptor tyrosine kinases and nuclear hormone receptors, and physically links E-cadherin to the actin cytoskeleton (53). Additional studies are needed to unravel the complex roles that individual Wnts have within the BMU and to determine the extent to which stabilized $\beta$-catenin can be used as a reliable surrogate for measuring Wnt activity.

Much remains to be learned about the molecular mechanisms of Wnt signaling, but it is clear that Wnts are potential targets for therapeutics designed to increase bone mass. One possible therapeutic approach would be to deliver Wnt agonists. The limitation of this approach, however, is that Wnts are historically very difficult and expensive to purify; thus, using Wnts as an anabolic agonist is impractical at this time. Efforts to therapeutically target the Wnt signaling pathway have instead focused on two alternative approaches (Figure 3): the first is to inhibit Wht antagonists (e.g. Dkk1, sclerostin, and Sfrp1) with neutralizing antibodies (discussed below), and the second is to inhibit glycogen syn- thase kinase $3 \beta$ (GSK3 $\beta$ ), which is a kinase that phosphorylates $\beta$-catenin and promotes its degradation. The latter approach has been accomplished in mice, whereby administration of lithium chloride and other small-molecule inhibitors of GSK $3 \beta$ reversed bone loss caused by aging, estrogen deficiency, and $\operatorname{Lrp} 5$ mutations (54); increased the sensitivity of osteoblasts and osteocytes to mechanical loading (30); and improved fracture healing (31). Lithium chloride is a commonly prescribed mood-stabilizing drug. Two studies examining fracture risk in humans found that lithium chloride use decreased fracture incidence in a dose-dependent manner (55); however, one study found that fracture incidence did not remain lower and actually increased after lithium chloride use was discontinued (56). Thus, the long-term effects of GSK3 $\beta$ inhibitors and other Wnt pathway agonists on bone formation and quality require careful evaluation before they can be used clinically as bone anabolic agents.

The other therapeutic approach being actively pursued to stimulate Wnt signaling to increase bone mass is the use of humanized monoclonal antibodies to neutralize antagonists of the Wnt signaling pathway. The canonical Wnt signaling pathway can be inhibited extracellularly by sequestering either the ligand or the receptor (Figure 3). Dkk1 and sclerostin inhibit Wnt signaling by dissociating LRP5 from Fz and Wnts. Sfrps, in contrast, bind Wnts and prevent them from associating with the LRP5/Fz complex. Serum Dkk1 levels are inversely proportional to bone mass in mice $(48,49,57)$ and are a prognostic biomarker of the osteolysis that is associated with multiple myeloma in humans (58). Similarly, Sfrp1 levels are inversely related to bone formation, but in mouse gene deletion studies, the results were more compelling in female mice than in male mice, indicating that Sfrp1 effects might be tied to hormonal influences (38). Sfrp1-specific neutralizing antibodies and antagonists have not yet been described. In contrast, Dkk1specific neutralizing antibodies have been tested in several studies $(59,60)$. Diarra and colleagues reversed bone destruction in a mouse model of rheumatoid arthritis by administering Dkk1-specific antibodies (59). This was accompanied by increased numbers of osteoblasts, rates of bone formation, and expression of OPG and by decreased numbers of osteoclasts. Interestingly, osteophytes (bone spurs) developed in the animals treated with Dkk1-specific antibodies, which effectively converted the phenotype to an osteoarthritic model. In a different study using a mouse model of multiple myeloma, a Dkk1-specific antibody promoted bone formation in tumor-bearing and non-tumor-bearing femurs (60). The Dkk1specific antibody reduced the number of osteoclasts expressing tartrate-resistant acid phosphatase (TRAP) and increased the number of osteoblasts producing osteocalcin. Testing of Dkk1-specific neutralizing antibodies is ongoing in preclinical animal models and these agents are likely to enter phase I clinical trials.

Although early results indicate that Dkk1-specific antibodies can promote bone formation, there are some concerns about their safety and potency. The development of osteophytes following administration of Dkk1-specific antibodies in the mouse model of rheumatoid arthritis suggests a role for Dkk1 in preventing osteoarthritis and bone spurs. However, optimization of dosage and delivery methods might control this outcome, and more studies are needed to define the appropriate doses and routes of delivery. Tumor formation is also a general concern, because the Wnt signaling pathway is activated by mutations in many cancers. However, it is well established that tumor formation requires multiple events; therefore, even though overstimulation of Wnt signaling 
pathways might contribute to tumorigenesis, it would be highly unlikely to be the sole initiating event. In anecdotal support of this, neither aged Dkk1 haploinsufficient mice nor humans using lithium chloride or with high bone mass due to gain-of-function mutations in LRP5 have increased cancer incidence (61).

\section{Targeting osteocyte function as a potential approach to enhancing bone anabolism}

Sclerostin is another promising target of biologic therapies to increase bone mass. Sclerostin is secreted exclusively by osteocytes, apparently as a mechanism to reduce bone formation $(62,63)$. Accordingly, sclerostin expression levels are repressed in response to mechanical loading and intermittent PTH treatment (64-66). Sclerostin is the product of the SOST gene, which is mutated and downregulated in patients with sclerosteosis and van Buchem disease $(67,68)$, diseases characterized by high bone density. Sclerostin inhibits osteoblast activity and bone formation by sequestering LRP5 and LRP6, thereby slowing the Wnt signaling pathway (Figure 3) (69). Interestingly, an LRP5 mutant that is associated with high bone mass prevents sclerostin from binding LRP5 (70), in both mice and humans. Sclerostin also binds BMPs, but with low affinity, nondiscriminately, and without directly affecting BMP2 target genes in osteoblasts $(62,63)$. Sclerostin thus seems to indirectly block BMP-induced bone formation by inhibiting Wnt signaling pathways. Preliminary studies with a humanized sclerostin-specific monoclonal antibody were promising and have shown bone anabolic activities in animal models and postmenopausal women $(71,72)$. Thus, inhibition of sclerostin activity seems to be a bone anabolic approach that is likely to progress rapidly toward clinical testing and ultimate clinical use.

\section{Targeting osteoblast-osteoclast cross-talk as a potential approach to enhancing bone anabolism}

Under most conditions, resorbed bone is nearly precisely replaced in location and amount by new bone. Thus, it has long been recognized that bone loss through osteoclast-mediated bone resorption and bone replacement through osteoblast-mediated bone formation are tightly coupled. There is now clear evidence that osteoblasts direct osteoclast differentiation by membrane presentation of RANKL and M-CSF $(73,74)$. The promotion of osteoclast differentiation by RANKL can be dampened through regulation of expression of the RANKL decoy receptor, OPG (74). In many instances, suppression of OPG, usually combined with increased expression of M-CSF and RANKL, enhances bone resorption (74). However, questions remain as to how osteoblasts are recruited to the site following the resorption phase and how the amount of bone laid down is controlled. This has led to consideration of how osteoclasts and/or their activity could promote bone formation.

Stored within the bone matrix are considerable quantities of several growth factors that could promote bone formation, such as TGF- $\beta$, IGF-I, and IGF-II $(75,76)$. Because osteoclast-mediated bone resorption releases IGFs and TGF- $\beta$ from bone, it has been posited that these play a part in coupling bone resorption to subsequent bone formation, although there is no direct evidence of this $(77,78)$. Examination of both mouse models and humans in whom osteoclastogenesis is perturbed has provided important insights into the role of bone resorption in promoting bone formation (79, 80 ). In mouse models where osteoclasts are present but not able to resorb bone (mice lacking either c-Src or chloride-7 channel), there is no defect in bone formation (81-83). Likewise, in humans who have one of their genes encoding the chloride-7 channel inactivated, osteoclasts are present, but there is inhibition of bone resorption with no reduction in bone formation (84-86). These data are in sharp contrast to mouse models that result in defective osteoclastogenesis, such as mice lacking c-fos or M-CSF, which have no osteoclasts and also exhibit defective bone formation (87-89). Thus, it seems that the presence of osteoclasts, whether or not they are resorbing bone, is needed for normal bone formation and that the release of bone-bound factors might not be required to couple osteoclasts to the bone formation phase of bone turnover.

What are possible osteoclast-osteoblast coupling factors? One intriguing recent discovery is the bidirectional signaling mediated by the transmembrane proteins ephrinB2 and EPH receptor B4 (EphB4), which are expressed on osteoclasts and osteoblasts, respectively (90). The engagement of these molecules stimulates osteoblast differentiation and represses osteoclast differentiation. A potential scenario is that osteoclast ephrinB2 stimulates osteoblast differentiation by binding EphB4 on osteoprogenitor cells, promoting bone formation. Conversely, EphB4 on osteoblastic cells binding ephrinB2 on osteoclast precursors represses further osteoclast differentiation. These interactions require close physical contact of osteoblast lineage cells with preosteoclasts and mature osteoclasts and are likely to occur in the BRC, as depicted in Figure $1 \mathrm{D}$. Indeed, the close proximity of osteoblast lineage cells to preosteoclasts and mature osteoclasts has already been demonstrated by the finding that membrane-bound RANKL and M-CSF on osteoblast lineage cells promote osteoclast differentiation (91, 92). There is evidence that osteoclasts might also recruit osteoblast lineage cells. Ryu et al. (93) have shown that sphingosine 1-phosphate (S1P), which is produced by osteoclasts, stimulates osteoblast migration, as well as promoting osteoblast survival. Thus, osteoclasts might recruit osteoprogenitors to sites of bone resorption by secretion of S1P and stimulate osteoblast differentiation by stimulating EphB4 signaling. In turn, osteoblast-stimulated reverse signaling through EphB4 binding to ephrinB2 on osteoclast precursors would repress osteoclast differentiation. This sequence of events would shut down bone resorption and set the stage for the next phase of bone remodeling, when osteoblasts are recruited to the site to replace the resorbed bone. Interest is growing in whether this stage of the bone remodeling cycle could provide novel anabolic targets.

\section{PTH uses multiple pathways to promote bone formation}

Although an excess of PTH, as in severe hyperparathyroidism, is often catabolic for bone, Fuller Albright observed almost 80 years ago that PTH can also have anabolic effects on bone (94). This led ultimately to a clinical trial clearly demonstrating that intermittent exposure of bone to PTH can increase bone formation and bone mass in humans (95). This is in marked contrast to continuous PTH exposure, which often leads to bone loss (96). Even though PTH is now approved by the FDA as a bone anabolic agent for patients with osteoporosis, determining the precise mechanism(s) by which it exerts its anabolic effects on bone still remains an area of intensive investigation. Emerging evidence indicates that part of the difficulty in unequivocally defining the mechanism(s) for the anabolic effects of PTH is that these effects probably involve multiple cell types and pathways. Thus, as summarized in Figure 4 and discussed below, PTH probably impacts osteoblasts, bone-lining cells, osteocytes, and osteoclasts and activates multiple pathways that collectively result in stimulation of bone formation. This 


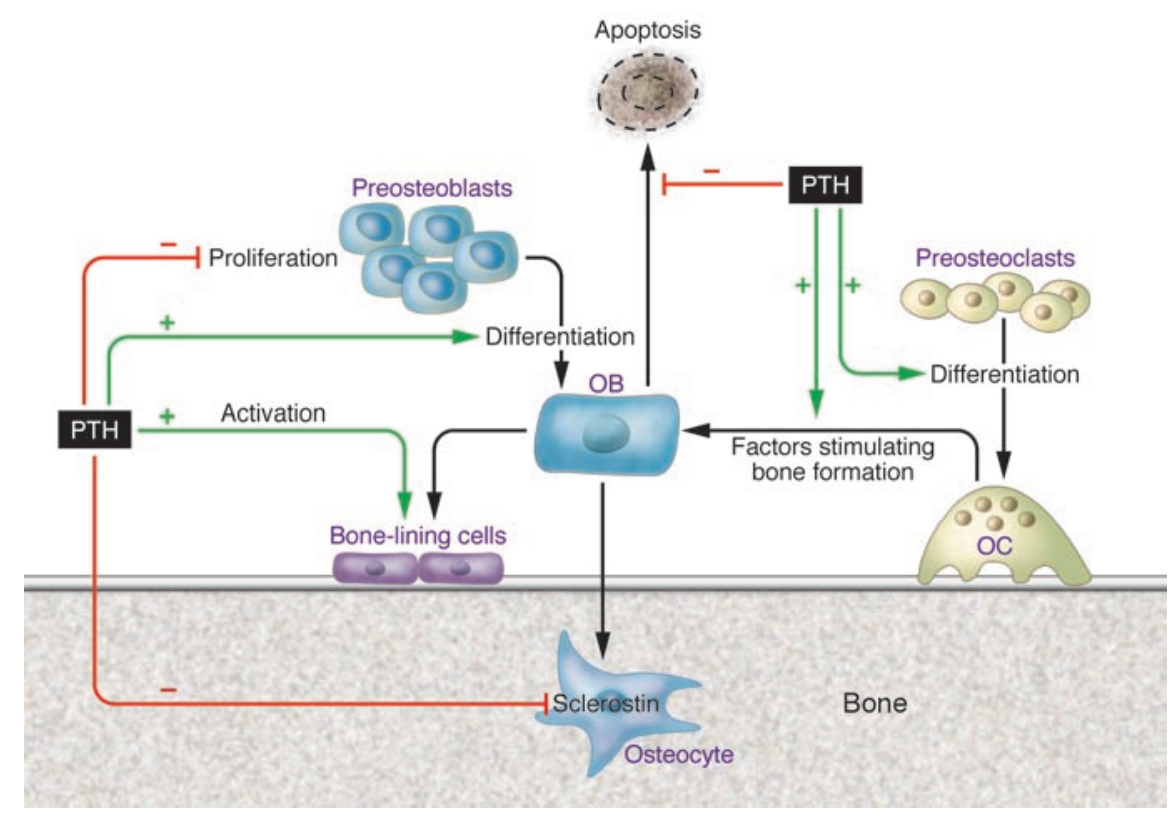

\section{Figure 4}

Potential cellular targets for the anabolic effects of PTH. PTH targets multiple cell types to mediate its bone anabolic effects. Specifically, PTH decreases apoptosis and proliferation of osteoblasts (OBs), as well as increasing their differentiation. It can activate bone-lining cells into functioning osteoblasts and decrease sclerostin production by osteocytes, which would be expected to increase Wnt signaling to osteoblasts. Finally, PTH is postulated to stimulate factors produced by osteoclasts (OCs) that stimulate osteoblasts. complexity has recently been demonstrated in microarray analyses of bones harvested from rats treated with $\mathrm{PTH}$, where a large number of genes $(\sim 1,000)$ were modulated by PTH $(97)$. These included genes involved in Wnt signaling (Wnt4 and Sfrp4), transcription factors (cAMP responsive element modulator [CREM]), growth factors (amphiregulin [AREG]), and chemokines (CCL2, also known as MCP-1]), and understanding how theses genes and gene regulatory networks ultimately result in an anabolic effect on bone is clearly an ongoing challenge.

As recently summarized by Jilka (98), the osteoblast is clearly a target for PTH action. Studies in mice have demonstrated that intermittent PTH treatment inhibits osteoblast apoptosis, at least in cancellous bone, leading to the suggestion that this might account, in large part, for the increase in osteoblast numbers and subsequent increase in bone formation seen following PTH therapy $(99,100)$. However, inhibition of osteoblast apoptosis by PTH is yet to be confirmed in humans (101); this might, in part, be due to the much lower basal prevalence of osteoblast apoptosis in mice versus humans (98). In terms of the specific mechanisms by which apoptosis is inhibited, studies in cultured osteoblastic cells have shown that PTH rapidly activates antiapoptotic signaling pathways that involve cAMP-mediated activation of PKA and its subsequent phosphorylation and inactivation of the proapoptotic protein BCL2-antagonist of cell death (Bad), as well as increased expression of survival genes such as B cell leukemia/lymphoma 2 (Bcl-2) (100). PTH also seems to decrease osteoblast proliferation, leading to a reciprocal increase in osteoblast differentiation (102); these effects might be due to decreased expression of cyclin D1, which is required for cell cycle progression, as well as increased expression of cyclin-dependent kinase inhibitors $(102,103)$. Concomitantly, PTH transiently increases the level and activity of Runx2 (100), and there are data supporting a role for LRP5-mediated Wnt signaling in mediating at least part of the anabolic effect of PTH (104). Some of the effects of PTH on osteoblastic cells might also be due to increased production and subsequent autocrine/paracrine effects of IGF-I and FGF-2 $(105,106)$. In addition, there is also evidence that PTH can "activate" previously quiescent bone-lining cells on bone surfaces (107), which might explain the very early increase in bone formation seen following PTH therapy. In contrast to these effects on differentiated osteoblastic cells, PTH does not seem to influence the proliferation or differentiation of uncommitted osteoprogenitor cells (99), in part because the PTH receptor is not expressed until these cells have progressed down the osteoblast lineage (108).

As noted earlier, until recently, the osteocyte was regarded as a relatively inert, quiescent cell entrapped in the bone matrix, but there is increasing evidence of a key role for the osteocyte in the regulation of bone remodeling (9). Consistent with this role, PTH has been shown to cause a transient reduction in the level of mRNA encoding sclerostin in osteocytes (65). This would, in turn, lead to increased Wnt signaling in the local microenvironment, and this might be an important mechanism by which PTH regulates osteoblast development via actions on the osteocyte.

Prior to the approval of PTH as a therapy for osteoporosis, there were a number of FDA-approved drugs, such as alendronate, that inhibited bone resorption. Since PTH stimulates bone formation, a logical hypothesis was that the simultaneous administration of PTH with an anticatabolic drug would lead to even larger increases in bone mass than administration of either agent alone. Surprisingly, however, this has proven not to be the case: studies in both women (109) and men (110) show that alendronate in fact blunts the anabolic action of PTH, at least in cancellous bone. This clinical observation - combined with animal work showing that absence of c-fos, which leads to a block in osteoclast differentiation, also results in loss of the anabolic response to PTH (111) - has led to the plausible hypothesis that at least part of the anabolic actions of PTH on bone are mediated by its activation of the osteoclast (79). Indeed, as discussed earlier, there is intense interest in identifying the factor(s) produced by osteoclasts that stimulate bone formation by osteoblasts.

\section{The future of bone anabolic therapies}

It is clear that within the closed compartment constituting the $\mathrm{BRC}$, there is an intimate cross-talk among osteoblasts, osteo- 
clasts, and bone-lining cells forming the canopy of the BRC. Moreover, the connections between the bone-lining canopy cells and bone-lining cells on the bone surface, which communicate with osteocytes, also allow the osteocytes embedded deep within the bone matrix to influence the numbers and activity of the osteoblasts. Understanding better these complex cellular interactions and the molecules that mediate them, such as those discussed in this Review, will ultimately lead to the development of new compounds, such as the sclerostin-specific antibody discussed above, with potent bone anabolic effects. This would, in turn, fulfill an important clinical need, since, although there are numerous compounds available that inhibit bone resorption, treatment options for reversing bone loss with bone formationstimulating agents are currently limited to PTH. Interestingly, it is somewhat ironic that despite the tremendous advances in basic bone biology that might lead to targeted anabolic therapies, PTH, which was discovered empirically to have anabolic effects on bone, continues to remain somewhat of a mystery in terms of its specific mechanisms of action.

\section{Acknowledgments}

This work was supported by NIH grants AG004875, AG028936, AR048147, AR050074, DE14680, and AR048147.

Address correspondence to: Sundeep Khosla, Guggenheim 7, College of Medicine, Mayo Clinic, 200 First Street SW, Rochester, Minnesota 55905, USA. Phone: (507) 255-6663; Fax: (507) 284-9111; E-mail: khosla.sundeep@mayo.edu.
1. Vahle, J.L., et al. 2002. Skeletal changes in rats given daily subcutaneous injections of recombinant human parathyroid human (1-34) for 2 years and relevance to human safety. Toxicol. Pathol. 30:312-321.

2. Rodriguez-Merchan, E.C., and Forriol, F. 2004. Nonunion: general principles and experimental data. Clin. Orthop. Rel. Res. 419:4-12.

3. Marsh, D. 1998. Concepts of fracture union, delayed union, and nonunion. Clin. Orthop. Rel. Res. 355(Suppl.):S22-S30

4. Owen, M. 1980. The origin of bone cells in the postnatal organism. Arthritis Rheum. 23:1073-1077.

5. Parfitt, A.M. 2000. The mechanism of coupling: role for the vasculature. Bone. 26:319-323.

6. Burkhardt, R., et al. 1984. The structural relationship of bone forming and endothelial cells of the bone marrow. In Bone Circulation. J. Arlet, R.P. Ficat, and D.S. Hungerford, editors. Williams \& Wilkins. Baltimore, Maryland, USA. 2-14.

7. Hauge, E.M., Qvesel, D., Eriksen, E.F., Mosekilde, L., and Melsen, F. 2001. Cancellous bone remodeling occurs in specialized compartments lined by cells expressing osteoblastic markers. J. Bone Miner Res. 16:1575-1582.

8. Everts, V., et al. 2002. The bone lining cell: its role in cleaning Howship's lacunae and initiating bone formation. J. Bone Miner. Res. 17:77-90.

9. Bonewald, L.F. 2007. Osteocyte messages from a bony tomb. Cell Metab. 5:410-411.

10. Eriksen, E.F., Eghbali-Fatourechi, G.Z., and Khosla, S. 2007. Remodeling and vascular spaces in bone. J. Bone Miner. Res. 22:1-6.

11. Wang, Y., et al. 2007. The hypoxia-inducible factor alpha pathway couples angiogenesis to osteogenesis during skeletal development. J. Clin. Invest. 117:1616-1626.

12. Brandi, M.L., and Collin-Osdoby, P. 2006. Perspective: Vascular biology and the skeleton. J. Bone Miner. Res. 21:183-192.

13. Iwaniec, U.T., et al. 2007. A comparative study of the bone-restorative efficacy of anabolic agents in aged ovariectomized rates. Osteoporos. Int. 18:351-362.

14. Li, M., et al. 2003. A novel, non-prostanoid EP2 receptor-selective prostaglandin E2 agonist stimulates local bone formation and enhances fracture healing. J. Bone Miner. Res. 18:2033-2042.

15. Qin, L., et al. 2003. Gene expression profiles and transcription factors involved in parathyroid hormone signaling in osteoblasts revealed by microarray and bioinformatics. J. Biol. Chem. 278:19723-19731.

16. Onyia, J.E., et al. 2005. Molecular profile of catabolic versus anabolic treatment regimens of parathyroid hormone (PTH) in rat bone: an analysis by DNA microarray. J. Cell. Biochem. 95:403-418.

17. Bodine, P.V.N., Seestaller-Wehr, L., Kharode, Y.P., Bex, F.J., and Komm, B.S. 2007. Bone anabolic effects of parathyroid hormone are blunted by deletion of the Wnt antagonist secreted frizzled- related protein-1. J. Cell Physiol. 210:352-357.

18. Westendorf, J.J., Kahler, R.A., and Schroeder, T.M. 2004. Wnt signaling in osteoblasts and bone diseases. Gene. 341:19-39.

19. Mbalaviele, G., et al. 2005. Beta-catenin and BMP-2 synergize to promote osteoblast differentiation and new bone formation. J. Cell. Biochem. 94:403-418.

20. Komori, T., et al. 1997. Targeted disruption of Cbfa1 results in a complete lack of bone formation owing to maturational arrest of osteoblasts. Cell. 89:755-764.

21. Otto, F., et al. 1997. Cbfa1, a candidate gene for cleidocranial dysplasia syndrome, is essential for osteoblast differentiation and bone development. Cell. 89:765-771.

22. Otto, F., Lubbert, M., and Stock, M. 2003. Upstream and downstream targets of RUNX proteins. J. Cell. Biochem. 89:9-18.

23. Lian, J.B., et al. 2006. Networks and hubs for the transcriptional control of osteoblastogenesis. Rev. Endocr. Metab. Disord. 7:1-16.

24. Nakashima, K., et al. 2002. The novel zinc fingercontaining transcription factor osterix is required for osteoblast differentiation and bone formation. Cell. 108:17-29.

25. Qu, G., and von Schroeder, H.P. 2006. Role of osterix in endothelin-1-induces downregulation of vascular endothelial growth factor in osteoblastic cells. Bone. 38:21-29.

26. Meury, T., Verrier, S., and Alini, M. 2006. Human endothelial cells inhibit BMSC differentiation into mature osteoblasts in vitro by interfering with osterix expression. J. Cell. Biochem. 98:992-1006.

27. Murshed, M., Harmey, D., Millan, J.L., McKee, M.D., and Karsenty, G. 2005. Unique coexpression in osteoblasts of broadly expressed genes accounts for the spatial restriction of ECM mineralization to bone. Genes Dev. 19:1093-1104.

28. Bonewald, L. 2006. Osteocytes as multifunctional cells. J. Musculoskelet. Neuronal Interact. 6:331-333.

29. Hens, J.R., et al. 2005. TOPGAL mice show that the canonical Wnt signaling pathway is active during bone development and growth and is activated by mechanical loading in vitro. J. Bone Miner. Res. 20:1103-1113.

30. Robinson, J.A., et al. 2006. Wnt/beta-catenin signaling is a normal physiological response to mechanical leading in bone. J. Biol. Chem. 281:31720-31828.

31. Chen, Y., et al. 2007. Beta-catenin signaling plays a disparate role in different phases of fracture repair: implications for therapy to improve bone healing. PLoS Med. 4:1216-1229.

32. Spencer, G.J., Utting, J.C., Etheridge, S.L., Arnett, T.R., and Genever, P.G. 2006. Wnt signalling in osteoblasts regulates expression of the receptor activator of NFkappaB ligand and inhibits osteoclastogenesis in vitro. J. Cell Sci. 119:1283-1296.

33. Gong, Y., et al. 2001. LDL receptor-related protein 5 (LRP5) affects bone accrual and eye development.
Cell. 107:513-523.

34. Little, R.D., et al. 2002. A mutation in the LDL receptor-related protein 5 gene results in the autosomal dominant high-bone-mass trait. Am. J. Hum. Genet. 70:11-19.

35. Kato, M., et al. 2002. Cbfa1-independent decrease in osteoblast proliferation, osteopenia, and persistent embryonic eye vascularization in mice deficient in Lrp5, a Wnt coreceptor. J. Cell Biol. 157:303-314.

36. Babij, P., et al. 2003. High bone mass in mice expressing a mutant LRP5 gene. J. Bone Miner. Res. 18:960-974.

37. Almeida, M., Han, L., Bellido, T., Manolagas, S.C., and Kousteni, S. 2005. Wnt proteins prevent apoptosis of both uncommitted osteoblast progenitors and differentiated osteoblasts by beta-catenindependent and -independent signaling cascades involving $\mathrm{Src} / \mathrm{ERK}$ and phosphatidylinositol 3-kinase/AKT. J. Biol. Chem. 280:41342-41351.

38. Bodine, P.V.N., et al. 2004. The Wnt antagonist secreted frizzled-related protein- 1 is a negative regulator of trabecular bone formation in adult mice. Mol. Endocrinol. 18:1222-1237.

39. Bodine, P.V.N., et al. 2005. The Wnt antagonist secreted frizzled-related protein-1 controls osteoblast and osteocyte apoptosis. J. Cell. Biochem. 96:1212-1230.

40. Bennett, C.N., et al. 2005. Regulation of osteoblastogenesis and bone mass by Wnt10b. Proc. Natl. Acad. Sci. U. S. A. 102:3324-3329.

41. Glass, D.A., II, et al. 2005. Canonical Wnt signaling in differentiated osteoblasts controls osteoclast differentiation. Dev. Cell. 8:751-764.

42. Hill, T.P., Spater, D., Taket, M.M., Birchmeier, W., and Hartmann, C. 2005. Canonical Wnt/betacatenin signaling prevents osteoblasts from differentiating into chondrocytes. Dev. Cell. 8:727-738.

43. Hu, H., et al. 2005. Sequential roles of hedgehog and Wnt signaling in osteoblast development. Development. 132:49-60.

44. Tamamura, Y., et al. 2005. Developmental regulation of Wnt/beta-catenin signals is required for growth plate assembly, cartilage integrity, and endochondral ossification. J. Biol. Chem. 280:19185-19195.

45. Day, T.F., Guo, X., Garrett-Beal, L., and Yang, Y. 2005. Wnt/beta-catenin signaling in mesenchymal progenitors controls osteoblast and chondrocyte differentiation during vertebrate skeletogenesis. Dev. Cell. 8:739-750.

46. Liu, H., et al. 2007. Augmented Wnt signaling in a mammalian model of accelerated aging. Science. 317:803-806.

47. Nakanishi, R., et al. 2006. Secreted frizzled-related protein 4 is a negative regulator of peak BMD in SAMP6 mice. J. Bone Miner. Res. 21:1713-1721.

48. Li, J., et al. 2006. Dkk1-mediated inhibition of Wnt signaling in bone results in osteopenia. Bone. 39:754-766. 
49. Morvan, F., et al. 2006. Deletion of a single allele of the Dkk1 gene leads to an increase in bone formation and bone mass. J. Bone Miner. Res. 21:934-945.

50. Rodda, S.J., and McMahon, A.P. 2006. Distinct roles for hedgehog and canonical Wnt in specification, differentiation and maintenance of osteoblast progenitors. Development. 133:3231-3244.

51. Kang, S., et al. 2007. Wnt signaling stimulates osteoblastogenesis of mesenchymal precursors by suppressing CCAAT/enhancer-binding protein alpha and peroxisome proliferator-activated receptor gamma. J. Biol. Chem. 282:14515-14524.

52. Boland, G.M., Perkins, G., Hall, D.J., and Tuan, R.S. 2004. Wnt 3a promotes proliferation and suppresses osteogenic differentiation of adult human mesenchymal stem cells. J. Cell. Biochem. 93:1210-1230.

53. McCrea, P.D., Turck, C.W., and Gumbiner, B. 1991. A homolog of the armadillo protein in drosophila (plakoglobin)associated with E-cadherin. Science. 254:1359-1361.

54. Clement-Lacroix, P., et al. 2005. Lrp5-independent activation of Wnt signaling by lithium chloride increases bone formation and bone mass in mice. Proc. Natl. Acad. Sci. U. S. A. 102:17406-17411.

55. Vestergaard, P., Rejnmark, L., and Mosekilde, L. 2005. Reduced relative risk of fractures among users of lithium. Calcif. Tissue Int. 77:1-8.

56. Wilting, I., et al. 2007. Lithium use and the risk of fractures. Bone. 40:1252-1258.

57. Macdonald, B.T., et al. 2007. Bone mass is inversely proportional to Dkk1 levels in mice. Bone. 41:331-339.

58. Tian, E., et al. 2003. The role of the Wnt-signaling antagonist DKK1 in the development of osteolytic lesions in multiple myeloma. N. Engl. J. Med. 349:2483-2494.

59. Diarra, D., et al. 2007. Dickkopf-1 is a master regulator of joint remodeling. Nat. Med. 13:156-163.

60. Yaccoby, S., et al. 2007. Antibody-based inhibition of DKK1 suppresses tumor-induced bone resorption and multiple myelome growth in vivo. Blood. 109:2106-2111.

61. Baron, R., and Rawadi, G. 2007. Targeting the Wnt/ beta-catenin pathway to regulate bone formation in the adult skeleton. Endocrinology. 148:2635-2643.

62. van Bezooijen, R.L., et al. 2007. Wnt but not BMP signaling is involved in the inhibitory action of sclerostin on BMP-stimulated bone formation. J. Bone Miner. Res. 22:19-28.

63. Winkler, D.G., et al. 2003. Osteocyte control of bone formation via sclerostin, a novel BMP antagonist. EMBO J. 22:6267-6276.

64. Keller, H., and Kneissel, M. 2005. SOST is a target gene for PTH in bone. Bone. 37:148-158.

65. Bellido, T., et al. 2005. Chronic elevation of parathyroid hormone in mice reduces expression of sclerostin by osteocytes: a novel mechanism for hormonal control of osteoblastogenesis. Endocrinology. 146:4577-4583.

66. Robling, A.G., Bellido, T., and Turner, C.H. 2006. Mechanical stimulation in vivo reduces osteocyte expression of sclerostin. J. Musculoskelet. Neuronal. Interact. 6:354.

67. Balemans, W., et al. 2001. Increase bone density in sclerosteosis is due to the deficiency of a novel secreted protein (SOST). Hum. Mol. Genet. 10:537-543.

68. Brunkow, M.E., et al. 2001. Bone dysplasia sclerosteosis results from loss of the SOST gene product, a novel cystine knot-containing protein. Am. J. Hum Genet. 68:577-589.

69. $\mathrm{Li}, \mathrm{X}$., et al. 2005. Dkk2 has a role in terminal osteoblast differentiation and mineralized matrix formation. Nat. Genet. 37:945-952

70. Ellies, D.L., et al. 2006. Bone density ligand, sclerostin, directly interacts with LRP5 but not LRP$5 \mathrm{G} 171 \mathrm{~V}$ to modulate Wnt activity. J. Bone Miner. Res. 21:1738-1749.
71. Ominsky, M.S., et al. 2006. Sclerostin monoclonal antibody treatment increases bone strength in osteopenic ovariectomized rats. J. Bone Miner. Res. 21:S44.

72. Padhi, D., et al. 2007. Anti-sclerostin antibody increases markers of bone formation in healthy postmenopausal women [abstract]. J. Bone Miner. Res. 22(Suppl. 1):S37.

73. Suda, T., Kobayashi, K., Jimi, E., Udagawa, N., and Takahashi, N. 2001. The molecular basis of osteoclast differentiation and activation. Novartis Found. Symp. 232:235-247; discussion 247-250.

74. Khosla, S. 2001. Minireview: the OPG/RANKL/ RANK system. Endocrinology. 142:5050-5055.

75. Dallas, S.L., Rosser, J.L., Mundy, G.R., and Bonewald, L.F. 2002. Proteolysis of latent transforming growth factor-beta (TGF-beta)-binding protein-1 by osteoclasts. A cellular mechanism for release of TGF-beta from bone matrix. J. Biol. Chem. 277:21352-21360.

76. Linkhart, T.A., and MacCharles, D.C. 1992. Interleukin-1 stimulates release of insulin-like growth factor-I from neonatal mouse calvaria by a prostaglandin synthesis-dependent mechanism. Endocrinology. 131:2297-2305.

77. Mohan, S., and Baylink, D.J. 1996. Insulin-like growth factor system components and the coupling of bone formation to resorption. Horm. Res. 45(Suppl.):59-62.

78. Bonewald, L.F., and Mundy, G.R. 1990. Role of transforming growth factor-beta in bone remodeling. Clin. Orthop. Relat. Res. 250:261-276.

79. Martin, T.J., and Sims, N.A. 2005. Osteoclastderived activity in the coupling of bone formation to resorption. Trends Mol. Med. 11:76-81.

80. Karsdal, M.A., Martin, T.J., Bollerslev, J., Christiansen, C., and Henriksen, K. 2007. Are nonresorbing osteoclasts sources of bone anabolic activity? J. Bone Miner. Res. 22:487-494

81. Marzia, M., et al. 2000. Decreased c-Src expression enhances osteoblast differentiation and bone formation. J. Cell Biol. 151:311-320.

82. Kornak, U., et al. 2001. Loss of the CiC-7 chloride channel leads to osteopetrosis in mice and man. Cell. 104:205-215.

83. Soriano, P., Montgomery, C., Geske, R., and Bradley, A. 1991. Targeted disruption of the c-src proto-oncogene leads to osteopetrosis in mice. Cell. 64:693-702.

84. Bollerslev, J., Steiniche, T., Melsen, F., and Mosekilde, L. 1989. Structural and histomorphometric studies of iliac crest trabecular and cortical bone in autosomal dominant osteopetrosis: a study of two radiological types. Bone. 10:19-24.

85. Atlatalo, S.L., et al. 2004. Osteoclast-derived serum tartrate-resistant acid phosphatase $5 \mathrm{~b}$ in AlbersSchonberg disease (type II autosomal dominant osteopetrosis). Clin. Chem. 50:883-890.

86. Del Fattore, A., et al. 2006. Clinical, genetic, and cellular analysis of 49 osteopetrotic patients: implications for diagnosis and treatment. J. Med. Genet. 43:315-325.

87. Dai, X.M., Zong, X.H., Akhter, M.P., and Stanley, E.R. 2004. Osteoclast deficiency results in disorganized matrix, reduced mineralization, and abnormal osteoblast behavior in developing bone. J. Bone Miner. Res. 19:1441-1451.

88. Sakagami, N., et al. 2005. Reduced osteoblastic population and defective mineralization in osteopetrotic (op/op) mice. Micron. 36:688-695.

89. Tuukkanen, J., et al. 2000. Mineral density and bone strength are dissociated in long bones of rat osteopetrotic mutations. J. Bone Miner. Res. 15:1905-1911.

90. Zhao, C., et al. 2006. Bidirectional ephrinB2-EphB4 signaling controls bone homeostasis. Cell Metab. 4:111-121.

91. Yasuda, H., et al. 1998. Osteoclast differentiation factor is a ligand for osteoprotegerin osteoclastogenesis-inhibitory factor and is identical to TRANCE/ RANKL. Proc. Natl. Acad. Sci. U. S. A. 95:3597-3602.

92. Lacey, D.L., et al. 1998. Osteoprotegerin ligand is a cytokine that regulates osteoclast differentiation and activation. Cell. 93:165-176.

93. Ryu, J., et al. 2006. Sphingosine 1-phosphate as a regulator of osteoclast differentiation and osteoclast-osteoblast coupling. EMBO J. 25:5840-5851.

94. Bauer, W., Aub, J.C., and Albright, F. 1929. Studies of calcium and phosphorus metabolism. J. Exp. Med. 49:145-161.

95. Neer, R.M., et al. 2001. Effect of parathyroid hormone (1-34) on fractures and bone mineral density in postmenopausal women with osteoporosis. N. Engl. J. Med. 344:1434-1441.

96. Tam, C.S., Heersche, J.N.M., Murray, T.M., and Parsons, J.A. 1982. Parathyroid hormone stimulates the bone apposition rate independently of its resorptive action: differential effects of intermittent and continuous administration. Endocrinology. 110:506-512.

97. Li, X., et al. 2007. Determination of dual effects of parathyroid hormone on skeletal gene expression in vivo by microarray and network analysis. J. Biol. Chem. 282:33086-33097.

98. Jilka, R.L. 2007. Molecular and cellular mechanisms of the anabolic effect of intermittent PTH. Bone. 40:1434-1446.

99. Jilka, R.L., et al. 1999. Increased bone formation by prevention of osteoblast apoptosis with parathyroid hormone. J. Clin. Invest. 104:439-446.

100.Bellido, T., et al. 2003. Proteasomal degradation of Runx 2 shortens parathyroid hormone-induced anti-apoptotic signaling in osteoblasts. J. Biol. Chem. 278:50259-50272.

101.Lindsay, R., et al. 2007. Effects if a one-month treatment with $\mathrm{PTH}(1-34)$ on bone formation on cancellous, endocortical, and periosteal surfaces of the human ilium. J. Bone Miner. Res. 22:495-502.

102.Qin, L., Li, X., Ko, J.K., and Partridge, N.C. 2005. Parathyroid hormone uses multiple mechanisms to arrest the cell cycle progression of osteoblastic cells from G1 to S phase. J. Biol. Chem. 280:3104-3111.

103.Datta, N.S., Chen, C., Berry, J.E., and McCauley, L.K. 2005. PTHrP signaling targets cyclin D1 and induces osteoblastic cell growth arrest. J. Bone Miner. Res. 20:1051-1064.

104. Kharode, Y.P., et al. 2006. Selective involvement of WNT signaling pathway in bone anabolic action of parathyroid hormone. J. Bone Miner. Res. 21:S114.

105. Bikle, D.D., et al. 2002. Insulin-like growth factor I is required for the anabolic actions of parathyroid hormone on mouse bone. J. Bone Miner. Res. 17:1570-1578.

106.Hurley, M.M., et al. 1999. Parathyroid hormone regulates the expression of fibroblast growth factor- 2 mRNA in osteoblastic cells. J. Bone Miner. Res. 14:776-783.

107. Dobnig, H., and Turner, R.T. 1995. Evidence that intermittent treatment with parathyroid hormone increases bone formation in adult rats by activation of bone lining cells. Endocrinology. 136:3632-3638.

108.Gronthos, S., et al. 1999. Differential cell surface expression of the STRO-1 and alkaline phosphatase antigens on discrete developmental stages in primary cultures of human bone cells. J. Bone Miner. Res. 14:47-56.

109.Black, D.M., et al. 2003. The effects of parathyroid hormone and alendronate alone or in combination in postmenopausal osteoporosis. N. Engl. J. Med. 349:1207-1215

110.Finkelstein, J.S., et al. 2003. The effects of parathyroid hormone, alendronate, or both in men with osteoporosis. N. Engl. J. Med. 349:1216-1226.

111.Koh, A.J., et al. 2005. Cells of the osteoclast lineage as mediators of the anabolic actions of parathyroid hormone in bone. Endocrinology. 146:4584-4596. 